ISSN: 0210-1696

DOI: http://dx.doi.org/10.14201/scero2015463725

\title{
AUTODETERMINACIÓN PERSONAL Y DISCAPACIDAD INTELECTUAL: UN ANÁLISIS DESDE LA PERSPECTIVA DE LAS FAMILIAS
}

\section{Self-Determination and Intellectual Disabilities: An Analysis from the Perspective of Families}

\author{
Araceli Arellano Torres \\ Departamento de Aprendizaje y Currículum. Facultad de Educación y Psicología. Universidad \\ de Navarra. Edificio de Biblioteca Antigua. Campus Universitario. 31080 Pamplona, Navarra \\ aarellanot@unav.es
}

Feli Peralta López

Departamento de Aprendizaje y Currículum. Facultad de Educación y Psicología. Universidad de Navarra

Recepción: 25 de mayo de 2015

Fecha de aceptación definitiva: 21 de julio de 2015

Biblid. [0210-1696 (2015) vol. 46 (3), n. ${ }^{\circ} 255$, julio-septiembre; 7-25]

Resumen: El contexto familiar juega un papel fundamental como apoyo a la autodeterminación de las personas con discapacidad intelectual; si bien la investigación al respecto es todavía escasa. En este artículo se presenta un estudio descriptivo que aborda la perspectiva de un grupo de padres y madres sobre la autodeterminación de sus hijos $(n=40)$. Se utilizó una entrevista semiestructurada que permitió valorar algunos conocimientos, actitudes y estrategias que pueden facilitar, o limitar, este derecho. Las familias de este estudio desconocen en su mayoría el concepto de autodeterminación, si bien valoran la autonomía e independencia como objetivos educativos prioritarios. Además, se muestran inseguras respecto a cómo apoyarles, muestran miedos variados y perciben a sus hijos como necesitados de protección. Sin embargo, también ponen en práctica algunas estrategias que favorecen la autodeterminación como ofrecer oportunidades para elegir o hablar acerca de sus características personales. Diversos factores relacionados con los rasgos propios de cada familia, sus necesidades, dinámicas diarias o ciclo vital, impactan en su percepción sobre esta meta. Se constata, por tanto, la importancia de 
comprender las experiencias de las familias para responder a sus necesidades y apoyarlas como facilitadoras de autodeterminación e inclusión social de sus hijos con discapacidad intelectual.

Palabras Clave: autonomía; inclusión social; padres; autodeterminación.

Aвstract: Family context plays an important role in supporting self-determination of people with intellectual disabilities. However, research regarding this topic is still limited. This article presents a descriptive study that addresses the perspective of a group of parents about their children's self-determination $(n=40)$. A semi-structured interview was used, in order to value certain knowledge, attitudes and strategies that can facilitate or limit self-determination skills. Families in this study mostly unknown the concept of self-determination, while value autonomy and independence as a priority educational objective. Also, they are insecure about how to support their children, and show fears. Besides, parents perceive their children as needed of protection. However, they also implemented some strategies that promote self-determination; such as providing opportunities to choose or talk about their personal characteristics. Several factors related to the distinctive features of each family, needs, or life cycle dynamics, impact on their perception of this goal. It is noted, therefore, the importance of understanding the experiences of families to meet their needs and support them as facilitators of self-determination and social inclusion of children with intellectual disabilities.

KEY WORDS: autonomy; social inclusion; parents; self-determination.

\section{Introducción}

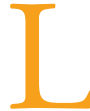

AS PERSONAS CON DISCAPACIDAD INTELECTUAL, tradicionalmente, han vivido sujetas a lo que terceras personas han considerado mejor para ellas. Con frecuencia, los educadores, las familias, los responsables de los servicios, entre otros, han decidido por ellas, elegido por ellas, evaluado sus necesidades, etc. De este modo, se podría decir que han sido educadas para la dependencia, y no tanto para la autonomía. Sin embargo, desde hace unas décadas se enfatiza el derecho de este colectivo a vivir su propia vida; a "emanciparse" de aquellos que han asumido roles de cuidado y (sobre)protección. El modelo socioecológico de la discapacidad, la defensa de la vida independiente o la propuesta sobre la diversidad funcional han propiciado este camino que lleva hacia el empoderamiento de las personas con discapacidad. El movimiento hacia la autodeterminación es, de hecho, uno de los mayores hitos en la defensa de los derechos de las personas con discapacidad intelectual (Ferguson, Ferguson y Wehmeyer, 2013; Verdugo et al., 2013; Wehmeyer y Little, 2013). Actualmente, es impensable hablar de buenas prácticas sin referirnos a este concepto, dado el consenso internacional en reconocerlo como dimensión de calidad de vida y meta fundamental del sistema de apoyos (Algozzine, Browder, Karvonen, Test y Wood, 2001; Verdugo y Schalock, 2009; Wehmeyer, Palmer, Shogren, WilliamsDiehm y Soukup, 2013). 
La autodeterminación y sus características personales y contextuales juegan un papel central y mediador en el logro de una vida plena y en la mejora de la calidad de vida personal. Varios estudios han mostrado la estrecha relación existente entre ambos constructos (Nota, Ferrari, Soresi y Wehmeyer, 2007; Lachapelle et al., 2005; Schalock, Bonham y Verdugo, 2008; Walker et al., 2011; Wehmeyer y Schalock, 2002; Wehmeyer et al., 2011). Desarrollar la autodeterminación supone incrementar la calidad de vida, ya que se pone el acento en la persona y en potenciar su autonomía e inclusión social, tomando en consideración sus intereses, preferencias, valores, metas y objetivos.

El desarrollo de la autodeterminación y el modo como se expresan las diferentes dimensiones de la conducta autodeterminada están condicionados por factores personales (edad, género, capacidad, etc.) y ambientales (cultura, contexto, escuela, familia, etc.) (Agran, Snow y Swaner, 1999; Peralta, González-Torres y Sobrino, 2005; Wehmeyer et al., 2011; Wehmeyer y Garner, 2003; Zhang, Landmark, Greenwelge y Montoya, 2010).

Precisamente, tomando como referencia el modelo socioecológico de la discapacidad, buena parte de la investigación se ha centrado en examinar aquellos factores ambientales que impulsan, u obstaculizan, la autodeterminación. Si bien, a este respecto, los datos sobre los contextos formales son abundantes (Wehmeyer et al., 2013; Wehmeyer y Field, 2007; Agran, Wehmeyer, Cavin y Palmer, 2008; Algozzine et al., 2001; Verdugo et al., 2013), carecemos de un conocimiento más profundo sobre los contextos familiares como facilitadores de autodeterminación (Burke, 2013; Curryer, Stancliffe y Dew, 2015; Shogren, 2013; Wehmeyer, 2014). A pesar de ello, la literatura coincide en resaltar la importancia del apoyo familiar para la adquisición de estas habilidades desde edades tempranas (Brotherson, Cook, Erwin y Weigel-Garrey, 2008; Wehmeyer, 2014; Burke, 2013; Palmer, 2010), siendo prácticamente imposible comprender la autodeterminación sin tomar en consideración su contexto familiar (Turnbull y Turnbull, 2000, 2001; Wehmeyer y Field, 2007).

Existen multitud de variables que determinan el modo en que la familia supone una barrera o un medio facilitador para promover la autodeterminación de sus hijos. Por ejemplo: percepciones y creencias de los padres respecto a la autodeterminación (Carter, Lane, Cooney, Weir, Moss y Machalicek, 2013; Zulueta y Peralta, 2008); background cultural y socioeconómico de la familia (Shogren, 2013; Zhang, Landmarck, Grenwelge y Montoya, 2010); o percepciones de control y habilidades de autodeterminación de los propios padres, así como su relación con los profesionales (Hardman, Drew y Egan, 2011; Wehmeyer y Field, 2007).

Por todo lo dicho, abordar este constructo desde un enfoque sistémico, centrado en la familia, es según Wehmeyer (2014: 183) una necesidad urgente. Es por ello que se precisa de un mayor cuerpo de datos acerca de cómo se vive la autodeterminación, desde la realidad de cada familia y no sólo como rasgo aislado del individuo (Shogren, 2013). 


\section{Objetivos}

Teniendo en cuenta este contexto, el propósito de este trabajo es presentar la perspectiva de un grupo de familias sobre la autodeterminación de sus hijos con discapacidad. Las preguntas específicas que guían este estudio son: ¿Qué visión tienen sobre la autodeterminación de sus hijos? ¿Qué estrategias utilizan para promover sus habilidades? ¿Qué obstáculos encuentran en este proceso?

\section{Método}

Los datos presentados forman parte de una investigación más amplia, que se llevó a cabo en dos fases (Arellano, 2012; Arellano y Peralta, 2013). En la primera, se trabajó con una muestra de 201 padres y madres que respondieron voluntariamente a un cuestionario elaborado ad boc. En la segunda fase, objeto de este artículo, se realizaron entrevistas a una parte de la muestra (que se prestó de manera voluntaria a colaborar), para indagar con mayor profundidad en sus experiencias concretas. La información de las próximas páginas está referida a esta segunda parte de la investigación.

\section{Procedimiento}

De la muestra total $(\mathrm{N}=201), 61$ personas accedieron a participar voluntariamente en esta segunda fase. Finalmente, fueron 42 los padres entrevistados, resultando 40 entrevistas válidas para el análisis. Las razones por las que se realizaron entrevistas a menos padres de los que accedieron a participar fueron variadas: sobre todo, relacionadas con circunstancias concretas de la familia que impedían mantener un encuentro con las investigadoras. Se eliminaron dos entrevistas por considerarlas no relevantes (respuestas excesivamente breves o falta de consentimiento para utilizar determinada información). En todos los encuentros mantenidos, se recordó el objetivo del estudio y se realizó la entrevista, según el protocolo explicado más adelante. Además de Pamplona (ciudad de pertenencia de las entidades que proporcionaron la muestra) fue necesario desplazarse hasta las localidades siguientes: Marcilla, Peralta, Olazagutía, Alsasua, Olatz, Barañáin, Los Arcos y Leiza (Navarra), y Arnedo (La Rioja).

\section{Participantes}

Los centros que proporcionaron la muestra para este estudio son: 2 centros de educación especial (Isterria y El Molino), el Centro de Orientación Familiar El Molino y la Asociación Síndrome de Down-Navarra (agradecemos a todos ellos su colaboración). Podemos suponer que la implicación de los padres en las entidades mencionadas, así como su participación voluntaria en el estudio, supone un sesgo importante de cara a la interpretación de resultados. La Tabla 1 recoge los datos 
significativos de la muestra, tanto de las personas entrevistadas como de sus hijos. En cuanto a la ocupación de los entrevistados, es complejo establecer categorías dada la heterogeneidad de las respuestas. Por lo tanto, quedan como siguen (solamente una persona deja sin responder esta cuestión): Jubilado/a 8; ama de casa 8; limpieza 1; educador/a 2; operaria 2 ; hostelería 1 ; pensionista 1 ; profesora 1 ; administrativa 1; enfermera 1; corredor de seguros 1 ; agricultora 1 ; agente comercial 1 ; arquitecto 1 ; cocinera 1 ; dependienta 1 ; camarera 1 ; economista 1 ; médico 1 ; modista 1 ; técnica en integración 1; celadora 1; prejubilado 1.

\begin{tabular}{|c|c|c|c|}
\hline \multicolumn{4}{|c|}{ Padres y madres } \\
\hline & Variable & $F r$ & $\%$ \\
\hline \multirow[t]{2}{*}{ Sexo } & Varón & 7 & $17.5 \%$ \\
\hline & Mujer & 33 & $82.5 \%$ \\
\hline \multirow[t]{4}{*}{ Edad } & $31-40$ & 1 & $2.5 \%$ \\
\hline & $41-50$ & 17 & $42.5 \%$ \\
\hline & $51-60$ & 12 & $30 \%$ \\
\hline & 61 o más & 10 & $25 \%$ \\
\hline \multirow[t]{5}{*}{ N. ${ }^{\circ}$ hijos } & 1 & 9 & $22.5 \%$ \\
\hline & 2 & 20 & $50 \%$ \\
\hline & 3 & 7 & $17.5 \%$ \\
\hline & 4 & 2 & $5 \%$ \\
\hline & 5 & 2 & $5 \%$ \\
\hline \multicolumn{4}{|c|}{ Personas con discapacidad } \\
\hline & Variable & $F r$ & $\%$ \\
\hline \multirow[t]{2}{*}{ Sexo } & Varón & 22 & $55 \%$ \\
\hline & Mujer & 18 & $45 \%$ \\
\hline \multirow[t]{3}{*}{ Edad } & $11-15$ & 10 & $25 \%$ \\
\hline & $16-20$ & 10 & $25 \%$ \\
\hline & 21 o más & 20 & $50 \%$ \\
\hline \multirow[t]{4}{*}{ Posición } & Es hijo único & 9 & $22.5 \%$ \\
\hline & Es el mayor & 13 & $32.5 \%$ \\
\hline & Es el pequeño & 10 & $25 \%$ \\
\hline & Otro & 8 & $20 \%$ \\
\hline
\end{tabular}

(C) Ediciones Universidad de Salamanca 


\begin{tabular}{|l|c|c|c|}
\hline \multicolumn{4}{|c|}{ Personas con discapacidad } \\
\hline Grado de DI & Ligero & 6 & $15 \%$ \\
\hline & Moderado & 14 & $35 \%$ \\
\hline & Severo & 17 & $42.5 \%$ \\
\hline & Profundo & 1 & $2.5 \%$ \\
\hline Lugar de residencia & Lo desconoce & 2 & $5 \%$ \\
\hline & Domicilio familiar & 32 & $80 \%$ \\
\hline & Piso independiente & 2 & $5 \%$ \\
\hline & Residencia/Centro & 6 & $15 \%$ \\
\hline
\end{tabular}

\section{Instrumento}

Aunque los datos sociodemográficos se recogieron durante la primera fase del estudio (mediante un cuestionario), en esta fase de la investigación se utilizó una entrevista semiestructurada. El guion de dicha entrevista se elaboró tomando como punto de partida el modelo funcional de autodeterminación de Wehmeyer (Wehmeyer et al., 2011; Wehmeyer, 2009; Wehmeyer y Field, 2007) así como otros instrumentos utilizados en investigaciones previas, de carácter similar (Erwin et al., 2009; Zulueta y Peralta, 2008; Zhang, 2005). La entrevista abordó seis categorías de información: (1) Conocimiento sobre autodeterminación, (2) Metas de la persona con discapacidad, (3) Toma de decisiones y elecciones, (4) Autoconocimiento y autoestima, (5) Riesgo y protección en los estilos educativos y (6) Expectativas sobre la autonomía. Se recoge en el Anexo 1 el protocolo utilizado.

\section{Análisis de datos}

Todas las entrevistas fueron grabadas, transcritas y formateadas para su tratamiento cualitativo, apoyándonos en el programa Aquad 6 (versión 2008). Para la codificación, adoptamos como unidad de registro el tema (el conjunto de afirmaciones que comparten un núcleo temático). Por otra parte, se decidieron las reglas de enumeración: la presencia o ausencia de los temas, la frecuencia y orden en que aparecen e intensidad con que son tratados. En cuanto a la categorización, los diferentes apartados de la entrevista aportaron las categorías principales de contenido y, a partir de ahí, se procedió a clasificar las respuestas de los padres a los temas planteados. Se combinaron procesos deductivos e inductivos y se utilizó el método comparativo como principal recurso, así como el procedimiento de triangulación de investigadores para interpretar un mismo cuerpo de datos. 


\section{Resultados y discusión}

De modo general, los resultados obtenidos son acordes con los de estudios previos, en cuanto a la importancia de la autodeterminación como meta a promover por las familias; las oportunidades que el contexto familiar ofrece para el aprendizaje de ciertas habilidades; así como las barreras que los padres encuentran en el camino hacia la autonomía de sus hijos (Zhang et al., 2010; Zulueta y Peralta, 2008; Zhang, 2005). Dichos resultados se presentan, a continuación, de acuerdo con las categorías prefijadas en la entrevista e incluyen indicadores de baja inferencia (citas literales de los padres) que ilustran el análisis de datos.

\section{Conocimiento sobre autodeterminación}

Las respuestas de los padres ante la pregunta ¿Qué entiende por autodeterminación? reflejan su desconocimiento generalizado acerca de dicho concepto. $\mathrm{Al}$ pedirles que definieran tanto la autodeterminación como la autogestión, los entrevistados adoptan tres posturas: (a) un $25 \%$ no conoce ninguno de los dos términos; (b) un 35\% no conoce el concepto de autodeterminación pero sí autogestión; (c) un $40 \%$ ha oído hablar y es capaz de aportar alguna idea acerca de ambos términos, aunque de manera vaga en la mayoría de ocasiones. En cualquier caso, las ideas asociadas a la autodeterminación, y la frecuencia con que aparece cada una, son las siguientes: autonomía, independencia, control o hacer las cosas solo (18 menciones); decisiones y elecciones (11); llevar una vida adulta normalizada (3); tener opinión propia (3); eficacia en habilidades de la vida diaria (2); y otras ideas, como ser independiente en actividades de ocio o manejar dinero ( 7 menciones). Como se ve, los entrevistados valoran significativamente la autonomía y la capacidad para desempeñar actividades concretas, sin ayuda. Esta perspectiva de la autodeterminación (sinónimo de autosuficiencia), si bien no es del todo incorrecta, es insuficiente y limitada (Wehmeyer, 2005).

Por otro lado, las familias encuentran difícil comprender el carácter evolutivo de la autodeterminación o la posibilidad de ser enseñada y aprendida. Existe una tendencia, ya advertida por Allen-Leigh, Katz y Rangel-Eudave (2008), a entender la autodeterminación como una realidad dicotómica (se tiene o no se tiene), marcada por el déficit y las limitaciones de la persona, y no como un proceso.

Padre 11: Me suena raro. Mi hijo es muy difícil que llegue a tener alguna de esas palabras, por su propia discapacidad. Es todo lo que le falta a mi hijo: independencia, capacidad, autonomía. Yo ese sueño no lo tengo.

Únicamente encontramos una madre que define de manera acertada el concepto de autodeterminación, al menos en sus aspectos fundamentales descritos por la literatura (Wehmeyer et al., 2011; Brown y Brown, 2009; Burton, Morgan y Davidson, 2005). 
Madre 38: Quiere decir que la persona con discapacidad llegue a ser autónoma, tenga control sobre su vida, elija, tenga opinión, digamos que sea una persona con entidad propia en la sociedad. Que tiene derecho a decidir lo que quiere ser, lo que quiere hacer en su vida, cuál va a ser su proyecto y su manera de vivir, desde los amigos, hasta si quiere tener pareja o no, si quiere vivir independiente...

\section{Metas de la persona con discapacidad}

En esta segunda categoría, las preguntas se centraron en comprobar si los padres conocían los objetivos de sus hijos y en qué medida está presente la expresión de preferencias en el contexto del hogar (su hijo, ¿tiene gustos claros?, ¿ sabe lo que le gusta, lo que no?, ¿muestra sus preferencias?, ¿'habla de actividades que le gustaría hacer, lugares que le gustaría visitar, etc.?). De manera genérica, los padres se muestran inseguros respecto a si sus hijos se plantean metas en la vida; concretamente, un $8 \%$ las consideran, de hecho, irreales y alejadas de sus posibilidades (hablan más bien de fantasías).

Madre 32: Mi hijo dice muchas cosas... Trabajar nada, dice que él quiere mirar a los que trabajan, de encargado, de jefe, de presidente del gobierno... también que quiere el carnet de conducir, que se quiere casar, tener hijos... no conozco ningún síndrome de Down que lo haya hecho.

Sin embargo, un 38\% reconocen metas específicas en sus hijos: "Sí, lo tiene muy claro: su meta principal es seguir trabajando y estar con su novia” (Padre 38); "Quiere vivir fuera, vestir como le gusta, si algún día quiere alquilar un piso con otros dos, pues lo tendremos que aceptar, yo estoy abierto" (Padre 4).

En otros casos, los padres reconocen que son ellos quienes moldean los objetivos de vida de sus hijos, teniendo en cuenta circunstancias y variables diversas que tienen que ver con rasgos propios de la familia (situación socioeconómica, por ejemplo); con el ciclo de vida familiar (y, en consecuencia, con la edad de sus hijos); con sus dinámicas de interacción o bien con el resto de necesidades y demandas. Esto ocurre, sobre todo, en el grupo de la muestra que tiene hijos pequeños, menores de 15 años (un 25\%); hecho, por otra parte, comprensible, dado que sus hijos no cuentan aún con experiencias, habilidades y conocimientos suficientes para tomar decisiones que aseguren su bienestar.

En cualquier caso, se confirma la necesidad de transformar lo que pudieran ser más bien sueños irrealizables, en objetivos realistas, tratando de llegar a consensos entre la persona con discapacidad y sus padres, cuyos intereses no siempre coindicen (Ponce, 2010; Palmer y Wehmeyer, 2002).

Por otra parte, la expresión de preferencias o gustos por parte de sus hijos está presente desde edades tempranas y parece que no implica demasiada dificultad, a diferencia del establecimiento de metas y objetivos que se perciben como un proceso complejo y sistematizado. 
Padre 12: Sí, sí tiene gustos claros, por ejemplo, psicoballet, natación, maquillaje. Tiene objetivos inmediatos, en función de esas cosas que le gustan. En cuestión de su futuro, no.

En cuanto a los comentarios sobre los intereses de sus hijos, los entrevistados señalan que son muy limitados (el $73 \%$ reconoce que sus hijos son felices con placeres inmediatos, como comer, ser abrazado, manualidades, etc.); tienden a repetirse (carácter rutinario y poco flexible); y varían con la edad. Concretamente, a partir de la adolescencia, cobran importancia tres campos de interés (todos los padres con hijos adolescentes mencionan, al menos, una de estas tres áreas): (1) la vida independiente, (2) el trabajo u ocupación y (3) las relaciones sociales, que generan, a su vez, preocupaciones en los padres, al percibir una brecha entre los sueños de sus hijos y la realidad que viven día a día.

Madre 5: A nivel personal quiere unas cosas que no son posibles... Claro, quiere a su novio, quiere amigas de su edad, quiere salir. Una serie de cosas que a mí me parece que no puede; pero no sé cómo hacérselo comprender.

Ya otros estudios (Gardiner e Iarocci, 2012; Ibáñez-López y Mudarra-Sánchez, 2004) han constatado la dificultad, precisamente, de adultos y jóvenes con discapacidad intelectual para mantener relaciones y amistades estables, algo que sigue siendo un reto pendiente y que, por lo tanto, debería ser un área prioritaria de intervención.

\section{Elecciones y toma de decisiones en actividades de la vida diaria}

En este punto nos interesamos por cuestiones como: ¿qué eligen las personas con discapacidad, en su día a dia?, ¿qué eligen sus padres?, ¿de quién dependen las decisiones y elecciones habitualmente? El análisis de las entrevistas deja, ante todo, una idea: las personas con discapacidad intelectual siguen dependiendo excesivamente de otros y, cuando se les da la oportunidad de realizar elecciones, éstas casi siempre se aplican a opciones relativamente reducidas y vinculadas a las rutinas diarias (Curryer et al., 2015; Palomo y Tamarit, 2000; Wehmeyer y Garner, 2003). Aun así, un 68\% de los padres afirman dejar elegir a sus hijos en áreas concretas: comida, bebida, actividades de ocio, ropa, etc. El $32 \%$ restante reconoce que sus hijos no eligen porque no saben, porque no se muestran seguros de sus decisiones o bien porque necesitan una supervisión constante (Madre 7: "No elige, no sabe, no le gusta, siempre elige lo mismo para comer, le da igual...”).

Durante la infancia y la adolescencia, los padres dicen ser ellos quienes toman aquellas decisiones importantes (por ejemplo, escolarización) pero que, aun así, están atentos a las preferencias de sus hijos. Por otra parte, les dan a elegir en áreas como la comida, las actividades de ocio o la ropa. El recurso más utilizado es plantear opciones (“¿quieres albóndigas o macarrones?”), siendo menos frecuentes las preguntas abiertas (“¿qué quieres comer?”). Cuanto más severa es la discapacidad, menos oportunidades 
para realizar elecciones (Wehmeyer, 2005). En estos casos, los padres cambian de estrategias: se centran en observar las reacciones de sus hijos.

Padre 11: Le notamos que tiene preferencia por ciertas cosas porque se las come con más deleite. Pero no le pregunto directamente ¿quieres fresas o uvas?

Las razones principales para estos padres, que justifican la ausencia de oportunidades para elegir, contrastadas también en otras investigaciones (Erwin et al., 2009; Zulueta y Peralta, 2008) son: la falta de tiempo, la lentitud de su hijo para elegir, su falta de motivación e interés, su gusto por las rutinas o el miedo a que elija mal. También existen algunas actitudes de los padres que pueden estar actuando como barreras y que convierten a la familia en un contexto un tanto restringido y poco facilitador de experiencias novedosas para la persona con discapacidad intelectual. Concretamente, creen que sus hijos son muy metódicos, rutinarios o incluso obsesivos, algo que alimenta la falta de oportunidades para elegir. Por otra parte, los padres afirman que conocen a fondo a sus hijos y no consideran importante que sean ellos quienes elijan o tomen la iniciativa ("ya sé lo que le gusta”). Brown y Brown (2009) ya advertían de que no informar a la persona con discapacidad, bajo la razón de que se conocen todas sus preferencias, es un error frecuente en el contexto familiar; tanto es así que Gómez y Cardona (2010) señalan esta indiferencia hacia los deseos de la persona como una forma concreta de discriminación en dicho contexto. Los datos recogidos en este estudio advierten de la necesidad de apoyar a las familias en la reflexión acerca de las razones que generan tan escasas oportunidades para la realización de elecciones.

Como era de esperar, tal y como señala la investigación (Burton et al., 2005; Turnbull y Turnbull, 2000, 2001), existen diferencias según el grado de discapacidad y la edad. La heterogeneidad de nuestra muestra permite conocer casos totalmente opuestos, en cuanto a su "poder" de decisión, aunque dificulta la elaboración de conclusiones definitivas. En ese sentido, están presentes experiencias muy variadas: por un lado, adultos que viven independientes y mantienen relaciones de pareja estables y, por otro, personas que no han adquirido hábitos de autocuidado básicos y presentan necesidades de apoyos extensos y generalizados.

Madre 16: Ha vivido solo, sus metas son trabajar, tener dinero, también se ha casado...

Madre 7: Ella no tiene capacidad de decisión. Tiene unos derechos, como todos, pero ¿puede ejercerlos? ¿O necesita una persona detrás? Mi hija es que necesita de mí las 24 horas del día...

En el caso de discapacidades severas, se confirma la creencia de algunos padres según la cual a mayores limitaciones menor importancia tiene la autodeterminación. Al contrario de lo que señala la investigación (Gómez-Vela, Verdugo, González, Badía y Wehmeyer, 2012; Wehmeyer et al., 2011; Chambers et al., 2007), para los padres el CI predice el nivel de autodeterminación y, por tanto, determina una futura vida de dependencia y falta de control. En repetidas ocasiones, algunos entrevistados señalan 
dar prioridad a otro tipo de valores, por encima de la autodeterminación, renunciando así a dar oportunidades para elegir a sus hijos. Por tanto, se constata que no se puede comprender la visión de los padres al margen de sus valores, rutinas, dinámicas y costumbres, tal y como señalan Turnbull y Turnbull (2001).

Madre 9: No le dejo elegir porque no tengo tiempo ni paciencia. Y porque valoro más el que nos vayamos de paseo que el que esté toda la mañana vistiéndose sola. O valoro más ir a la piscina y estar un rato con gente, que estar dos horas preparándose la mochila.

\section{Autonocimiento y autoestima}

Dada la importancia del autoconocimiento como elemento de la autodeterminación, es interesante conocer qué peso conceden a la discapacidad como un rasgo de sus hijos. En esta parte de las entrevistas, abundan los silencios, las frases dubitativas o las expresiones como "No sabría definirlo; Es difícil explicar cómo es", etc.

La mayoría de padres menciona la discapacidad como uno de los rasgos definitorios de su hijo, aludiendo incluso al nivel de minusvalía o al diagnóstico ("Tiene un $85 \%$ de discapacidad"). Muchos se sirven de esta etiqueta para describirlos de un modo rápido. De alguna manera, les resulta difícil ir más allá de las limitaciones de sus hijos, lo cual nos advierte del posible efecto halo de la discapacidad (García de la Cruz, 2009). Sin embargo, guiando a los padres con preguntas variadas (¿qué cosas buenas tiene su hijo?, ¿qué se le da bien?, ¿qué es lo que más le gusta de su bijo?, ¿y lo que menos?), éstos definen a sus hijos de una manera más completa, en términos de cualidades ("noble, alegre, feliz, contento, sincero, comprensivo, ordenado, educado") y de rasgos negativos ("rebelde, vago, agresivo, machista, pasivo, pesado, desconfiado, manipulador, infantil”). La información recogida sobre este punto, además, ayuda a combatir la creencia de que las personas con discapacidad intelectual son todas iguales.

Además de la propia visión de las familias, un tema a tratar dentro de esta categoría es el conocimiento que la persona tiene sobre sí misma. Es por eso por lo que se les planteó a los padres la siguiente cuestión: ¿cree que su hijo sabe cómo es? (autoconocimiento). Un $60 \%$ afirma que su hijo conoce algunos de sus rasgos más característicos (físicos y de personalidad). Para el $20 \%$ su hijo no es consciente de sus características tanto físicas como psicológicas; es decir, que no sabrían responder a preguntas del tipo ¿de qué color tienes los ojos?, ¿qué cosas se te dan bien?, ¿cómo tienes el pelo? Esto ocurre, mayoritariamente, en los padres cuyos hijos tienen una discapacidad severa o son pequeños. El 20\% restante duda acerca de si su hijo alcanza este grado de autoconocimiento básico, reconoce no haber hablado nunca con sus hijos sobre este tema y muestra sorpresa al no poder responder con rotundidad a esta cuestión. Éste es un aspecto importante, dado que el autoconcepto parece ser una variable significativa que influye en el resto de dimensiones de calidad de vida.

En cuanto al conocimiento específico sobre la discapacidad ( $\dot{C}$ Cree que su hijo sabe, percibe que tiene una discapacidad?) se pueden agrupar las respuestas de los padres en tres categorías: (a) un 40\% afirma que sus hijos son conscientes de su discapacidad y, 
además, lo aceptan de manera natural; (b) un 25\% creen que, si bien son conscientes de su discapacidad, esto les genera cierto sufrimiento; y (c) un 35\% opina que sus hijos no son conscientes de su discapacidad. Comentarios como los siguientes ilustran todos estos casos:

Madre 37: Ella sabe que tiene una discapacidad, lo cual me parece maravilloso, porque hablamos de discapacidad delante de ella, se reconoce como persona discapacitada, se sabe diferente a los demás. Pero se ve querida, protegida y, yo creo, que de momento es feliz siendo como es.

Madre 18: Ahora está con que a ver por qué ha nacido él así, lleva como medio año preguntándome que a ver cuándo se va a curar. Yo le digo que no se cura, que él ha nacido así. A mí eso me llega al alma. Veo esa frustración.

Padre 11: Afortunadamente, no se da cuenta de que tiene una discapacidad. Eso debe ser tremendo, puede ser muy duro...

Aunque en buena parte los padres consideran importante y beneficioso que sus hijos se conozcan, algunos prefieren que sus hijos no sean conscientes de sus diferencias (de alguna manera, piensan, la ignorancia les protegerá de la inseguridad, frustración, temor que supone el verse como alguien diferente). Power (2008) advierte cómo, en ocasiones, los padres manifiestan una "negación” de la discapacidad de su hijo (no hablan de ella, no comentan el tema), a modo de estrategia de afrontamiento. Esta postura es una importante barrera para la inclusión social, dado que dificulta una visión natural de la diversidad humana. Es éste un problema complejo que lleva a pensar en la carga negativa que todavía tiene la etiqueta de "discapacidad". Estos enunciados parecen indicar que las personas con discapacidad y sus familias todavía enfrentan estigmas frecuentes que impactan en su bienestar y que deberían ser tenidos en cuenta en los programas y estrategias de intervención psicoeducativos y sociales (Ali, Hassiotis, Stydom y King, 2012; Wehmeyer, 2014; Bacon y O’Hara, 2011; Wehmeyer et al., 2011; Chambers et al., 2007). En la medida en que desaparezca dicho estigma, las familias se encontrarán más cómodas a la hora de tratar con naturalidad todo lo relacionado con las limitaciones de sus hijos y promover un autoconcepto saludable.

\section{Riesgo y protección en sus estilos educativos}

Ya se ha adelantado cómo, con frecuencia, el miedo al riesgo, ciertas normas culturales, actitudes de protección, etc., hacen que el entorno familiar sea un tanto restringido para el desarrollo de la autodeterminación (Brotherson et al., 2008). Una de las estrategias para promover la autodeterminación es establecer un equilibrio entre permitir arriesgarse y proteger. Con frecuencia, las familias no se sienten capacitadas (empoderadas) para asumir ciertos niveles de riesgo, a veces porque no encaja en su sistema de valores o necesidades. Se analizan a continuación las respuestas de los participantes a las preguntas relacionadas con este aspecto: ¿considera que le da la suficiente autonomía a su bijola?, ¿cree que tiende a sobreprotegerle? 
El 67\% de la muestra opina haber motivado a la persona con discapacidad a correr riesgos, experimentar, ser independiente, etc. En menor medida (33\%), reconocen haber mantenido actitudes sobreprotectoras, limitando así sus oportunidades para la autodeterminación. Así pues, la mayor parte de los padres recalcan el hecho de que, en este camino, siempre han estado acompañándoles y supervisando los riesgos. Aunque todos ellos han marcado límites, coinciden en haber sido flexibles y pacientes durante el proceso de aprendizaje.

Los factores que impulsan estilos educativos favorables a la autodeterminación, identificados a partir de los comentarios de los padres, son: valoración de la autodeterminación como una meta educativa que merece la pena, rasgos de personalidad de los padres (por ejemplo, conciencia sobre la importancia de establecer límites) o existencia de logros y mejoras que animan a los padres a seguir adelante. Es el caso de las siguientes madres:

Madre 25: Hemos intentado que tuviera una vida normal. Hay veces que igual ha dicho "voy a fregar" e igual tarda dos horas, pues en vez de decirle "quita, ya lo hago yo", le he dejado.

Madre 37: (...) monta a caballo y galopa como nadie, como una jinete. Y si tiene habilidad es porque, cuando yo aún tenía miedo, ella cizañaba al caballo para que corriera más.

En cualquier caso, independientemente de los logros conseguidos, el 99\% de los padres comparten una misma idea: aunque siempre han sentido miedos a la hora de "soltar" a sus hijos, no se han dejado vencer por ellos y han asumido un cierto riesgo.

Padre 14: [hablando sobre cómo darle autonomía a su hija]. Es como enseñar a andar en bicicleta a un hijo, le pones las ruedicas, le empujas... Pero llega un momento en que se las quitas, y le tienes que dejar solo. Y sabes que se puede caer, y probablemente se caiga, pero lo tienes que hacer. Hombre, le proteges, pues le pones un casco, unas rodilleras... Pero, si no le sueltas, no aprende.

En cuanto al grupo de padres que reconocen limitar las oportunidades de sus hijos para la autonomía, la independencia y la autorregulación (33\%), mencionan razones muy variadas, que tienen que ver con factores, ya señalados por autores como Friend y Burscuk (2012) o Wehmeyer y Field (2007). En primer lugar, razones relacionadas con sus propias actitudes y percepciones: miedo a peligros físicos fuera de casa (por ejemplo, el tráfico) y dentro de casa (por ejemplo, el fuego); falta de paciencia y tiempo para invertir en el aprendizaje de tareas; miedos específicos en áreas concretas (por ejemplo, sobre la sexualidad); valoración de otras metas por encima de la autodeterminación; desconocimiento sobre cómo aumentar la autodeterminación de sus hijos y sobre la implicación que eso tiene para sus vidas; etc. En segundo lugar, relacionadas con la persona con discapacidad intelectual: problemas de conducta; escasas habilidades comunicativas; falta de iniciativa; falta de responsabilidad; “manipulación” de su entorno (Madre 25: "Sé que puede pero no lo hace porque lo 
hago yo"); lentitud a la hora de realizar ciertas tareas, elegir, decidir, etc. A los padres les resulta complicado dejar que sus hijos vivan su propia vida, percibiéndolos como indefensos y poco capacitados para ello. Cuando las limitaciones son severas, las familias carecen de estrategias sobre cómo apoyarles a tener más control sobre aspectos de su día a día. A ello, como se ha dicho anteriormente, se suman, en el $20 \%$ de los entrevistados, miedos específicos acerca de posibles situaciones de maltrato o abuso. No en vano, la investigación pone de manifiesto la mayor incidencia de abusos, físicos y psicológicos, en niños y jóvenes con discapacidad, en comparación con aquellos sin discapacidad (Stalker y McArthur, 2012), dato que las familias parecen tener bien presente.

Madre 32: Tuvimos un problema gordo, se lo llevaron por ahí, no sabemos qué pasó, si le pegaron o pasó algo peor... Un día vino alterado y no sabemos por qué; solo que él decía que no le gustaba... se lo han hecho a él porque lo ven indefenso, como a un niño pequeño. El miedo ese ya no me lo quita nadie.

La distribución de las respuestas en los dos grupos mencionados (apoyo a la autonomía y sobreprotección) es heterogénea atendiendo a variables como el sexo del hijo o la edad. Únicamente, cuando la discapacidad es severa parecen concentrarse en el segundo grupo. No obstante, son más bien variables personales o características distintivas de cada familia las que pueden influir en los estilos educativos (percepción sobre la discapacidad, atribuciones de las dificultades de sus hijos, etc.) (Dempsey y Dunst, 2004).

\section{Expectativas sobre la autonomía de sus bijos}

Finalmente, en este estudio, el $95 \%$ de los participantes son optimistas sobre el futuro, aunque comedidos. Son conscientes de sus limitaciones y apelan a la prudencia de no aspirar a metas muy altas. Aun con todo ello, se muestran confiados en que sus hijos mejoren, siendo más positivas las expectativas en padres con hijos de menor edad. Así, un 33\% reconoce que sus hijos son menos autónomos en casa que en otros entornos (escolar, por ejemplo) de modo que confían en que "pueden dar más de sí”.

Los objetivos de los padres a este respecto también varían significativamente en función del nivel de discapacidad y, por tanto, del impacto que ésta tiene en las dinámicas, interacciones y rutinas familiares (Guyard et al., 2012). Aquellos con hijos con discapacidades ligeras mencionan como prioritarias la vida independiente y la adquisición de habilidades de manejo del hogar (como cocinar, planchar, comprar...). En el caso de los hijos con discapacidades moderadas, los objetivos se centran en aumentar la participación en la vida familiar y social (expresar mejor sus preferencias, habilidades sociales...). Finalmente, cuando sus hijos tienen discapacidades severas y profundas, los padres centran sus expectativas en el desarrollo de hábitos en actividades de la vida diaria (higiene o alimentación), así como en la mejora de ciertas conductas específicas (manejo de rabietas...). 
Únicamente dos entrevistados (de los 40) aseguran no tener esperanzas en que sus hijos sean más autodeterminados. Ambos consideran que sus hijos "han tocado techo” y lo único que les queda es, en todo caso, trabajar para no perder los aprendizajes adquiridos.

\section{Limitaciones}

Es preciso señalar las siguientes limitaciones del estudio: (a) la participación voluntaria de la muestra, el sesgo en edad y sexo; (b) el tamaño de la muestra; (c) el carácter local de la investigación, que dificulta la generalización de conclusiones; (d) el sesgo que conlleva el rol del investigador como herramienta de análisis de datos; (e) la finalidad puramente descriptiva del estudio, y (f) el empleo de los padres como informantes acerca de las conductas y percepciones de las personas con discapacidad. Dadas estas limitaciones, los datos deben tomarse como resultados descriptivos, que podrían ser completados en un futuro con las percepciones de las propias personas con discapacidad intelectual, dado que seguramente varíen respecto a las que tienen sus padres.

\section{Conclusiones}

Aunque la importancia de la familia como principal agente de autodeterminación es incuestionable, como pone de manifiesto la investigación consultada, son aún escasos los trabajos que ahonden en la perspectiva de los padres sobre este derecho y meta. Este estudio, a pesar de las limitaciones, ha permitido conocer de primera mano algunas de sus experiencias. Ha supuesto una oportunidad para dar voz a las familias, y hacerlas partícipes de los grandes cambios que a favor de la autodeterminación y del empoderamiento están teniendo lugar.

Las características de la familia como sistema, las dinámicas de interacción establecidas con la persona con discapacidad, el ciclo de vida por el que cada familia transita o el resto de demandas y necesidades presentes influyen en la perspectiva de los padres acerca de la autodeterminación, en la importancia que le otorgan como meta y en las oportunidades que ofrecen a sus hijos para ejercer este derecho. Así, de acuerdo con los datos obtenidos, las familias de este estudio, aunque han oído hablar de la autodeterminación (40\%), no conocen su significado y no comprenden del todo las implicaciones en la vida de sus hijos (60\%). Además, el 33\% encuentra barreras importantes a la hora de equilibrar riesgo y protección (miedos, falta de tiempo, otras necesidades más urgentes...) y necesitan apoyo para identificar las razones de por qué sus hijos no son más autodeterminados. Como consecuencia, estas personas con discapacidad cuentan aún con escasas oportunidades para ejercer el control y siguen dependiendo de otros para decidir qué hacer, cómo, cuándo y dónde, también en el contexto familiar.

Por otra parte, a pesar de las dificultades mostradas, entendemos que las familias encierran un gran potencial como contexto de apoyo a la autodeterminación. A lo 
largo de las entrevistas, se han identificado ciertos puntos fuertes que sustentan esta idea del entorno familiar como un medio privilegiado: (a) los padres valoran, en su mayoría, la autodeterminación como una de las metas más importantes en la vida de su hijo; (b) son quienes mejor conocen las preferencias, gustos e intereses de la persona con discapacidad; (c) ofrecen un marco de seguridad y afecto incondicional que protege a la persona de posibles fracasos y riesgos excesivos; (d) aun cuando tienden a sobreproteger, los padres se preocupan por conseguir cierto nivel de autonomía, y (e) otorgan la posibilidad de hacer elecciones y tomar decisiones en áreas cotidianas con las que la persona está habituada.

Ofrecer esta información a los profesionales de los servicios puede ser un medio para promover relaciones colaborativas con las familias, basadas en el respeto a sus valores, el reconocimiento de sus roles y la escucha activa. Dicha colaboración, entre los apoyos naturales y formales, es imprescindible de cara a mejorar la autonomía, la calidad de vida y la participación social de las personas con discapacidad intelectual.

\section{Referencias bibliográficas}

Agran, M., Wehmeyer, M. L., Cavin, M. y Palmer, S. (2008). Promoting student active classroom participation skills through instruction to promote self-regulated learning and self-determination. Career Development for Exceptional Individuals, 31, 106-114.

Algozzine, B., Browder, D., Karvonen, D., Test, W. y Wood, W. M. (2001). Effects on interventions to promote self-determination for individuals with disabilities. Review of Educational Research, 71, 219-277.

Bacon, A. y O'Hara, D. (2011). Self-determination across the life span: Issues and gaps. Exceptionality, 19, 31-45.

Brotherson, M. J., Cook, C. C., Erwin, E. J. y Weigel-Garrey, C. J. (2008). Understanding self-determination and families of young children with disabilities in home environments. Journal of Early Intervention, 31 (1), 22-43.

Brown, I. y Brown, R. (2009). Choice as an aspect of quality of life for people with intellectual disabilities. Journal of Policy and Practice in Intellectual Disabilities, 6 (1), 11-18.

Burke, M. (2013). Improving parental involvement: training special education advocates. Journal of Disability Policy Studies, 23 (4), 225-234.

Burton, R., Morgan, M. y Davidson, J. (2005). Does the daily choice making adults with intellectual disability meet the normalization principle? Journal of Intellectual and Developmental Disabilities, 30 (4), 226-235.

Chambers, C. R., Wehmeyer, M. L., Saito, Y., Lida, K. M., Lee, Y. y Singh, V. (2007). Selfdetermination: What do we know? Where do we go? Exceptionality, 15 (1), 3-15.

Curryer, B., Stancliffe, R. y Dew, A. (2015). Self-determination: adults with intellectual disability and their family. Journal of Intellectual and Developmental Disability [versión electrónica]. DOI: 10.3109/13668250.2015.1029883

Dempsey, I. y Dunst, C. J. (2004). Helpgiving styles and parent empowerment in families with a young child with a disability. Journal of Intellectual and Developmental Disability, 29 (1), 40-51.

Erwin, E. J., Brotherson, M. J., Palmer, S. B., Cook, C. C., Weigel-Garrey, C. Y. y Summers, J. A. (2009). How to promote self-determination for young children with 
disabilities: evidenced-based strategies for early childhood practitioners and families. Young Exceptional Children, 12 (2), 27-37.

Ferguson, D. L., Ferguson, P. M. y Wehmeyer, M .L. (2013). The self-advocacy movement: Intellectual disability in late modern times (1980-Present). En M. L. WeHMEYer (Ed.), The story of intellectual disability: An evolution of meaning, understanding, and public perception (pp. 237-282). Baltimore: Paul H. Brookes.

Friend, M. y Bursuck, W. D. (2012). Including students with special needs. A practical guide for classroom teachers (6. ${ }^{\mathrm{a}}$ ed.). Greensboro, NC: Pearson.

Gómez, J. M. y Cardona, M. C. (2010). Percepciones y actitudes de los padres acerca de la discriminación de sus hijos por razón de discapacidad intelectual. Educación y Diversidad, 4 (1), 73-88.

Gómez-Vela, M., Verdugo, M. Á., González, F., Badía, M. y Wehmeyer, M. L. (2012). Assessment of the self-determination of Spanish students with intellectual disabilities and other educational needs. Education and Training in Autism and Developmental Disabilities, 47 (1), 48-57.

Guyard, A., Michelsen, S., Arnaud, C., Lyons, A., Cans, C. y Fauconnier, J. (2012). Measuring the concept of impact of childhood disability on parents: validation of a multidimensional measurement in a cerebral palsy population. Research in Developmental Disabilities, 33 (5), 1594-1604.

Palmer, S. B. (2010). Self-determination: a life span perspective. Focus on Exceptional Children, $42,1-16$

Palmer, S. B. y Wehmeyer, M. L. (2002). A parent's guide to the self-determined learning model for early elementary students. Kansas: Beach Center on Disability.

Palomo, R. y Tamarit, J. (2000). Autodeterminación: analizando la elección. Siglo Cero, 31 (3), 21-41.

Ponce, A. (2010). Formación en autodeterminación para familias. Madrid: Confederación Española de Organizaciones en favor de las Personas con Discapacidad Intelectual (FEAPS).

Power, A. (2008). Caring for independent lives: Geographies of caring for young adults with intellectual disabilities. Social Science E Medicine, 67 (5), 834-843.

Shogren, K. A. (2013). A social-ecological analysis of the self-determination literature. Intellectual and Developmental Disabilities, 51 (6), 496-511.

Stalker, K. y McArthur, K. (2012). Child abuse, child protection and disabled children: a review of recent research. Child Abuse Review, 21 (1), 24-40.

Turnbull, A. P. y Turnbull, H. R. (2000). Fostering family-professional partnerships. En M. E. SNell y F. Brown (Eds.), Instruction of students with severe disabilities (5. $\left.{ }^{a} \mathrm{ed}.\right)$ (pp. 31-66). Nueva Jersey: Prentice Hall.

Turnbull, A. P. y Turnbull, H. R. (2001). Self-determination for individuals with significant cognitive disabilities and their families. Association for Persons with Severe Handicaps, 26 (1), 56-62.

Verdugo, M. Á., Gómez-Vela, M., Fernández, R., Vicente, E., Wehmeyer, M. L. y otros (2013). ¿Cómo evaluar la autodeterminación? Escala ARC-INICO de evaluación de la autodeterminación. Siglo Cero, 44 (4), 21-39.

Verdugo, M. Á. y SCHALOCK, R. L. (2009). Quality of life: from concept to future applications in the field of intellectual disabilities. Journal of Policy and Practice in Intellectual Disabilities, 6 (1), 62-64.

Wehmeyer, M. L. (2005). Self-determination and individuals with severe disabilities: Reexamining meanings and misinterpretations. Research and Practice for Persons with Severe Disabilities, 30 (3), 113-120. 
WehmeYer, M. L. (2009). Autodeterminación y la tercera generación de prácticas de inclusión. Revista de Educación, 349, 45-67.

Wehmeyer, M. L. (2014). Self-determination: a family affair. Family Relations. Interdisciplinary Journal of Applied Family Studies, 63 (1), 178-184.

Wehmeyer, M. L., Abery, B., Zhang, D., Ward, K., Willis, D., Amin, W. y otros (2011). A series of paper on scaling-up efforts to promote self-determination. Personal selfdetermination and moderating variables that impact efforts to promote self-determination. Exceptionality, 19, 19-30.

Wehmeyer, M. L. y Field, S. (2007). Self-determination: instructional and assessment strategies. Thousand, CA: Corwin Press.

Wehmeyer, M. L. y Garner, N. W. (2003). The impact of personal characteristics of people with intellectual disability on self-determination and autonomous functioning. Journal of Applied Research in Intellectual Disabilities, 16, 255-265.

Wehmeyer, M. L. y Little, T. D. (2013). Self-determination, positive psychology, and disability. En M. L. Wehmeyer (Ed.), Handbook of positive psychology and disability (pp. 116-136). Oxford: Oxford University Press.

Wehmeyer, M. L., Palmer, S., Shogren, K., Williams-Diehm, K. y Soukup, J. H. (2010). Establishing a causal relationship between intervention to promote self-determination and enhanced student self-determination. The Journal of Special Education, 4, 195-2010.

Zhang, D. (2005). Parent practices facilitating self-determination skills: The influences of culture, socioeconomic status and children's special education status. Research and Practice for Persons with Severe Disabilities, 30 (3), 154-162.

Zhang, D., Landmark, L., Greenwelge, C. y Montoya, C. L. (2010). Culturally diverse parents perspectives on self-determination. Education and Training in Autism and Developmental Disabilities, 45, 175-186. 


\section{ANEXO 1 \\ Protocolo de entrevista para padres y madres de personas con discapacidad intelectual}

\section{DATOS GENERALES}

Edad de la persona con discapacidad intelectual; hermanos; lugar de residencia; ocupación actual; etc.

\section{CONOCIMIENTO SOBRE AUTODETERMINACIÓN}

1. ¿Había oído hablar de la autodeterminación, en relación a su hijo/a con discapacidad?

(a) si es que sí, ¿podría explicar qué significa?

(b) si es que no, ¿ha oído hablar de autogestión o autodefensa?

2. ¿Cómo interpreta la siguiente afirmación?: “Mi hijo/a tiene derecho a la autodeterminación”.

\section{METAS DE SU HIJO/A CON DISCAPACIDAD}

3. ¿Cree que las personas con discapacidad tienen metas propias, objetivos en su vida? ¿Y su hijo/a? ¿Diría que sabe lo que quiere?

\section{RIESGO Y PROTECCIÓN}

4. ¿Considera que le da la suficiente autonomía a su hijo/a? ¿Le cuesta dejarle hacer cosas solo/a? ¿Tiende a sustituirle cuando hace mal una actividad? ¿Siente miedo al dejarle hacer cosas solo/a? ¿Piensa que le ha sobreprotegido?

\section{TOMA DE DECISIONES Y ELECCIONES}

6. Piense en su día a día con su hijo/a, ¿decide por él/ella muchas cosas a lo largo de sus actividades diarias? ¿Cómo participa su hijo/a en decisiones cotidianas? Si decide por él/ella ¿por qué cree que es?

\section{EXPECTATIVAS SOBRE AUTONOMÍA}

7. ¿Cree que su hijo/a podría ser más autónomo? ¿Le gustaría que así fuera?

\section{AUTOCONOCIMIENTO Y AUTOESTIMA}

8. ¿Cómo definiría a su hijo/a? ¿Qué cualidades tiene? ¿Habla con su hijo/a de sus cosas buenas? ¿Cree que su hijo/a sabe cómo es?

9. ¿Cree que su hijo/a sabe, se siente, percibe, que es diferente? ¿Se identifica con otras personas con discapacidad? ¿Cree que ha sufrido por ello en algún momento de su vida? ¿Le ha preguntado alguna vez las razones de sus dificultades? 